\title{
THE LUMINOSITY DETERMINATION
}

\section{Based on observations made with the ESA Hipparcos satellite}

\author{
A.E. GÓMEZ, C. TURON \\ DASGAL/URA 335 du CNRS, Observatoire de Paris-Meudon \\ 92195 Meudon Cedex, FRANCE \\ e-mail: anita@obspm.fr
}

\section{Introduction}

The Hertzprung-Russel (HR) diagram luminosity calibration relies basically on three kinds of data: trigonometric parallaxes, kinematical data (proper motions and radial velocities) and cluster distances obtained by the zeroage main sequence fitting procedure. The most fundamental method to calculate the absolute magnitude is the use of trigonometric parallaxes, but up to now, accurate data only exist for stars contained in a small volume around the sun. Individual absolute magnitudes are obtained using trigonometric parallaxes or photometric and spectroscopic calibrations. In these calibrations the accuracy on the absolute magnitude determination ranges from $\pm 0^{m} 2$ in the main sequence to $\pm 0^{m} 5$ in the giant branch. On the other hand, trigonometric parallaxes, kinematical data or cluster distances have been used to make statistical calibrations of the absolute magnitude. The standard error on the mean absolute magnitude calibrations ranges from $\pm 0^{m} 3$ to $\pm 0^{m} 6$ on the mean sequence, from $\pm 0^{m} 5$ to $\pm 0^{m} 7$ on the giant branch and is of about $1^{m}$ for supergiants.

Future improvements in the absolute magnitude determination will depend on the improvement of the basic data from the ground and space. A brief overview of the new available data is presented. In particular, the analysis of the first 30 months data of the Hipparcos mission (H30) (from the 37 months data of the whole mission) allows to perform a statistical evaluation of the improvements expected in the luminosity determination.

\section{New available data}

Table 1 gives the different sources of trigonometric parallaxes existing or to come in the near future. The columns are self-explanatory. $\mathrm{N}$ is the number 
of stars, $\mathrm{V}$ is the magnitude in the Johnson system and $\mathrm{H}$ is the magnitude corresponding to the large bandwidth of the Hipparcos detector. Comparing with the other sources, Hipparcos provides a larger number of high quality parallaxes and, in addition, its parallaxes are absolute. Hipparcos also provides proper motions for about 120000 stars with a mean expected accuracy of $1.5 \mathrm{mas} / \mathrm{yr}$, better than the announced nominal value.

TABLE 1. Trigonometric parallax data (expressed in milli-arcsec)

\begin{tabular}{lclcl}
\hline Data Source & $N$ & Limit mag. & Precision & Targets \\
\hline New Yale Cat. & $\mathbf{7 8 8 8}$ & $V \simeq 20$ & $1-25$ & in vanAltena et al, 92 \\
Ground-based & $<\mathbf{3 0 / \text { year }}$ & $V \simeq 20$ & $0.5-5$ & Cool dwarfs, cluster stars, etc. \\
HIPPARCOS & 100000 & $H=12.5$ & $1-5$ & Input Cat. (Turon et al, 92) \\
HUBBLE ST & a few & $V \simeq 17$ & 0.5 & Proxima,Barnard's *,PN central \\
& & & & stars,6 Hyades stars, variables,etc.
\end{tabular}

A large variety of stars were observed by Hipparcos: stars of all spectral types, peculiar stars, variable stars, binary stars and stars in open clusters (Gómez 1993). Byproducts of the astrometric mission are the determination of the H-magnitude with a precision of about a few milli-magnitudes and the discovery of new variables and new binaries (see Perryman 1994). Table 2 shows the mean distance modulus precision for the clusters closer than $250 \mathrm{pc}$, calculated using the formal errors on parallaxes of the H30 solution. The distances are taken from Mermilliod (1988); $N$ is the number of observed stars. Final Hipparcos results are foreseen for the end of 1996.

TABLE 2. Cluster stars: mean distance modulus precision in $\mathrm{H} 30$

\begin{tabular}{lrrc}
\hline Nom & $\mathbf{r}(\mathbf{p c})$ & \multicolumn{1}{c}{$N$} & $\epsilon\langle m-M\rangle_{0}$ \\
\hline Hyades & $\mathbf{4 8}$ & 209 & $\pm 0^{m} 02$ \\
Coma Ber & 80 & 117 & $\pm 0^{m} 06$ \\
Pleiades & 125 & 74 & $\pm 0^{m} 12$ \\
IC 2602 & 150 & 34 & $\pm 0^{m} 08$ \\
IC 2391 & 155 & 21 & $\pm 0^{m} 10$ \\
NGC 2632 & 160 & 22 & $\pm 0^{m} 22$ \\
$\alpha$ Per & 165 & 133 & $\pm 0^{m} 10$ \\
NGC 6475 & 240 & 12 & $\pm 0^{m} 35$ \\
Blanco 1 & 240 & 18 & $\pm 0^{m} 23$ \\
\hline
\end{tabular}

\section{The Luminosity Determination}

In less than three years we will be faced with an enormous amount of new high quality data allowing to review and improve the stellar luminosity determination. Preliminary H30 parallax data show that direct absolute magnitudes will be obtained in the range of $M_{V}-4$ to $14 \mathrm{mag}$, extending the luminosity determination to stars intrinsically more luminous than 
$\mathbf{M}_{V} \sim 0$ mag. Expected errors on absolute magnitudes due to the error in the parallax are: $\leq \pm 0^{m} 5$ for about 40000 stars (spectral types from M5 up to $\mathrm{BO}$ on the main sequence, the subgiant and the giant branches and a few supergiants); $\leq \pm 0{ }^{m} 2$ for about 15000 stars (spectral types up to B0 on the main sequence, 1000 giants); $\leq \pm 0^{m} 1$ for 3000 stars (2000 dwarfs up to B3, 200 giants), and $\leq \pm 0{ }^{m} 02$ for 200 stars (dwarfs up to A0 and a few giants and subgiants from F2 to K2). On the other hand, high precision fainter absolute magnitudes, up to $\mathrm{M}_{V} \sim 19$, are obtained from ground-based photoelectric astrometry (Monet et al. 1992)

In addition to the improvement in individual absolute magnitude determinations, high quality parallaxes and proper motions allow to improve the statistical luminosity calibrations: we expect about 60000 stars with parallax relative errors better than $30 \%$; 36000 stars with proper motions relative errors better than $5 \%$ and 82000 stars with proper motions relative errors better than $20 \%$. Moreover, direct distance determination of about 20 open clusters of various ages and metallicities will be available and this will make possible to check the classical zero-age main sequence fitting procedure. This amount of new data will require a careful definition of the samples as well as the improvement of the calibration methods. In order to illustrate the first point, we selected from the Inca Data Base a sample of $\mathrm{K} 2 \mathrm{III}$ stars on $\mathrm{H} 30$ parallaxes with relative errors better than $15 \%$ and calculated their absolute magnitudes. The results show a large scatter in $\mathbf{M}_{V}$, of about $3 \mathrm{mag}$, in contradiction with the usually accepted standard errors on the mean absolute magnitude calibrations for giants $\left( \pm 0{ }^{m} 5- \pm 0^{m} 7\right)$. The aim of the calibration methods is to obtain an unbiased estimator of the absolute magnitude. Recently, new methods based on maximum likelihood estimation have been developped (Ratnatunga \& Casertano 1991, Arenou 1994, Luri et al. 1994). These methods make a simultaneous determination of the absolute magnitude and its dispersion and, at the same time, correct from the sampling bias and take into account the observational errors on the data. They are well suited to be applied to incomplete samples.

\section{References}

Arenou, F. 1994, I.A.U. Symposium 166, to appear

Gómex, A. E., 1993, A.S.P. Conf. Ser. 40, 324

Luri, X., Mennessier, M.O., Torra, J. \& Figueras, F., $1994 A \& A$, in preparation

Mermilliod, J.C. 1988, Bull. Inform. CDS, 31, 175

Monet, D. et al. $1992, A_{J} 103,638$

Perryman M.A.C., 1994, I.A.U. Symposium 166, to appear

Ratnatunga, K.U. \& Casertano, S., 1991, AJ 101, 1075

Turon, C. et al. 1992 The Hipparcos Input Catalogue, ESA SP-1136.

vanAltenaW.F., Lee, J.T. \& Hoffleit, E.D., 1992, CD-ROM preliminary version 
E. GRIFFIN to R. CAYREL Q. This refers to the temperature of Arcturus, which you quoted at the beginning of your talk. In 1975, a careful analysis of the observed spectra of Arcturus concluded that the mass of Arcturus was low, too low for comfort of the HD. During the ensuing battle between the theoreticians and the observers, a temporary solution was found if the effective temperature, which was believed to be higher than that used by the observers. Now, however, the new value which you quote is only 9 degres away from the value used by the observers, and I invite you to comment on the implication for the theory of the H-R diagram.

A. If the effective temperature is only 9 degres away from that of the $\mathbf{1 9 7 5}$ paper, it is clear thay the problem was not with the temperature but with the gravity of the object. It is to the speaker in charge of the luminosity determination that should direct your question.

D.F. GRAY to R. CAYREL Q. The original line-depth ratio using VI $\lambda$ 6251.83 to FeI $\lambda 6252.57$ is metal dependent. This does not impair the use of the ratio for measuring temperature variations using rotational modulation or stellar cycles. A recent analysis of $\mathbf{1 0}$ new line-depth ratio will be published in the PASP. These are weak lines and avoid any metallicity effect.

C. VAN 'T VEER to R. CAYREL Q. I would add two things. First: computed $\mathrm{H} \alpha$ profiles become very sensitive to metallicity for overabundant models. Second: another convective parameter very important is the use or not of overshooting. $\mathrm{H} \alpha$ and following lines in the Balmer serie are strongly sensitive to this parameter. Then the use of $\mathrm{H} \alpha$ only can give a wrong $\mathrm{T}_{\text {eff }}$.

A. $\mathrm{H} \alpha$ is still the best Balmer line to use, but you are right in mentionning that the Balmer lines are pretty metal dependent, because of the poor treatement of convection in stellar atmosphere. At present there is no generally agreed way of testing overshooting in model atmosphere computations.

W. TOBIN to R. CAYREL Q. I'am surprised people continue to use infra-red flux method. In A.A 125, 168, 1983 I showed that what it does is compared the derredenned observed ratio (monochromatic flux/integrated flux) with values predicted by model atmosphere. There is no special advantage of infrared monochromatic fluxes because the flux level there is still contingent on correct opacities at all other wavelength.

(continued after the paper by Hubeny) 